\title{
EFEITO DO ENSACAMENTO DE FRUTOS SOBRE DANOS CAUSADOS POR FATORES BIÓTICOS E ABIÓTICOS EM POMAR ORGÂNICO DE MACIEIRA ${ }^{1}$
}

\author{
JANAÍNA PEREIRA DOS SANTOS² \& ANDERSON FERNANDO WAMSER ${ }^{2}$
}

\begin{abstract}
RESUMO - Este trabalho teve como objetivos avaliar o efeito do ensacamento dos frutos de macieira na incidência de danos causados por insetos-praga, sarna, "russeting" e queimadura pelo sol, além da praticidade dos diferentes tipos de embalagens utilizadas no ensacamento. O estudo foi conduzido no pomar orgânico de macieira da empresa Fischer Fraiburgo Agrícola Ltda, em Fraiburgo-SC, na safra 2004-2005. O delineamento experimental foi o de blocos casualizados. Os tratamentos foram: a) frutos não ensacados (testemunha), e b) frutos ensacados, seguido de desensacamento aos 15 e 7 dias antes da colheita, e na colheita. Ensacaram-se, por tratamento, 150 frutos da cultivar Royal Gala e 300 frutos da cultivar Suprema, utilizando-se de dois tipos de embalagens: saco de papel-manteiga parafinado branco e de polipropileno microperfurado transparente. Observou-se que o ensacamento reduziu o ataque de pragas em relação aos frutos não ensacados. Porém, a sarna desenvolveuse, tanto em frutos ensacados, quanto em não ensacados. Em geral, houve menos frutos com "russeting", com a antecipação da retirada dos sacos. A embalagem de polipropileno é mais fácil de ser manuseada, resiste à chuva e ao granizo, e apresentou menor custo que a de papel; entretanto, facilitou a queimadura de frutos pelo sol, principalmente quando se encontra muito aderida à epiderme destes.
\end{abstract}

Termos para indexação: Malus domestica, produção orgânica, fitossanidade.

\section{FRUIT BAGGING EFFECT ON THE DAMAGE CAUSED BY BIOTICS AND ABIOTICS FACTORS IN AN ORGANIC APPLE ORCHARD}

\begin{abstract}
This study had as objective the evaluation of fruit bagging effect on the damage incidence caused by insect pests, apple scab, russeting and sunburn on apple fruits, and the practicity of different bagging bags used in organic apple orchard. The study was carried out at Fischer Fraiburgo Agrícola Ltda, in Fraiburgo, SC. It was used a completely randomized block design. The treatments were applied as follows: a) no bagging fruits (control); b) bagging fruits, followed by the unbagging at 15 and 7 days before harvest, and on the harvest day. It was bagged per treatment, 150 'Royal Gala' fruits and 300 'Suprema' fruits, using white paraffinic paper bags or transparent polypropylene microperfurated bags. It was observed that bagging reduced the insect-pest attack in relation to the unbagged fruits. However, apple scab developed on bagged and unbagged fruits. Usually, anticipating the unbagging there was less "russeting" fruit. Bagging is easier with the polypropylene bags, the fruits are less damaged by rain and hail, and the bags are cheaper than the paper ones, however, it facilitated sunburn, mainly, when the bag is too adhered to the epidermis of these fruits.
\end{abstract}

Index terms: Malus domestica, organic production, phytossanitary.

\section{INTRODUÇÃO}

A maçã é a fruta de clima temperado de maior importância comercial como fruta fresca, tanto no contexto nacional, como no internacional (ABPM, 2003). No Brasil, na safra 2003-2004, a fruta obteve a maior produção da história, com 977.863 toneladas (IBGE, 2004). Concentrada na região Sul, cerca de $31 \%$ da produção é de 'Fuji' e 30\% de 'Gala', com expressivo crescimento dos clones melhorados, com aumento de $82 \%$ de clones de 'Fuji' na safra 20032004, em relação à de 2002-2003, e de 77\% dos clones de 'Gala', para este mesmo período (ABPM, 2004). A cultivar Royal Gala é uma mutação espontânea da 'Gala', introduzida comercialmente em 1974, apresentando frutos de coloração vermelho-estriada brilhante. A cultivar Suprema é uma mutação somática de um ramo de 'Fuji', apresenta frutos bastante vermelhos, sendo que esta coloração cobre mais de $80 \%$ da superfície do fruto (Camilo \& Denardi, 2002). Ambas as cultivares são muito suscetíveis ao ataque de insetos-praga, doenças, especialmente a sarna, e distúrbios fisiológicos. Estes fatores podem provocar depreciação na aparência dos frutos e modificar o sabor da polpa, reduzindo, conseqüentemente, o valor comercial da fruta in natura.

Segundo Boneti et al. (2002), a sarna, causada pelo fungo Venturia inaequalis, é a principal doença da macieira nas regiões de clima temperado e úmido, podendo ocasionar até $100 \%$ de perdas caso não sejam adotadas medidas de controle. Segundo Basso (2002), o "russeting" é um importante distúrbio fisiológico, por deixar áspera e de coloração ferruginosa a epiderme dos frutos, depreciando a classificação do produto e diminuindo a renda do produtor.

Nesta cultura, geralmente, o controle de insetos-praga e doenças é feito através da aplicação de agroquímicos em cobertura. Além de dispendiosos, estes produtos podem afetar a fauna de inimigos naturais, reduzir a diversidade biológica e provocar desequilíbrio ambiental. Entretanto, estes riscos podem ser diminuídos quando os frutos são ensacados. Faoro \& Yasunobu (2001) comentam que, quando frutos de pereira são ensacados, a incidência de pragas e doenças, conforme o ano, pode variar de 1,1 a 3,8\%. Segundo Faoro (2003), esta técnica vem sendo preconizada há muitos anos pela pesquisa e já é utilizada por alguns produtores de pêra, que visam a eliminar ou reduzir o uso de inseticidas e fungicidas. Faoro (2000) comenta que o ensacamento de frutos de pêra japonesa é realizado para evitar danos de pássaros, insetos-praga, doenças, "russeting", diminuir o número de aplicações e a quantidade de defensivos químicos, amenizar danos ocasionados por chuvas leves de granizo e melhorar a aparência dos frutos. De acordo com Telles et al. (2004), como na produção orgânica existe o impedimento do uso de inseticidas sintéticos, uma das opções seria o ensacamento dos frutos.

No sistema de produção orgânica de maçãs, o ensacamento dos frutos também pode ser uma alternativa para o controle de insetospraga e doenças, sem afetar a qualidade, preservando a aparência e o preço de mercado. Este trabalho teve como objetivo avaliar dois tipos de embalagens para o ensacamento de frutos e três épocas de desensacamento, sobre a incidência de danos causados pelos fatores bióticos: insetos-praga e sarna, e pelos abióticos: "russeting" e a queimadura pelo sol, nas cultivares de macieira Royal Gala e Suprema.

\footnotetext{
(Trabalho 155-2005). Recebido: 03-10-2005. Aceito para publicação: 19-05-2006.

2 Eng. Agr., M.Sc., EPAGRI, Estação Experimental de Caçador. Abílio Franco, C.P. 591, 89500-000, Caçador-SC, fone: (49) 3561 2035. E-mail: janapereira@ epagri.rct-sc.br.

2 Eng. Agr., M.Sc., EPAGRI, Estação Experimental de Caçador. Abílio Franco, C.P. 591, 89500-000, Caçador-SC, fone: (49) 3561 2023. E-mail: afwamser@epagri.rct-sc.br.

Órgão financiador: EMBRAPA/PRODETAB
} 
TABELA 1- Percentagem de frutos danificados por fatores bióticos, em função do tipo de embalagem utilizada no ensacamento e da época de desensacamento - cv. Suprema. Fraiburgo-SC, safra 2004-2005.

\begin{tabular}{|c|c|c|c|c|c|c|c|c|}
\hline \multirow[b]{2}{*}{ Tratamento $^{1}$} & \multicolumn{8}{|c|}{ Danos avaliados (\%) } \\
\hline & $\begin{array}{c}\text { Mariposa } \\
\text { oriental }\end{array}$ & $\begin{array}{c}\text { Lagarta- } \\
\text { Enroladeira }\end{array}$ & $\begin{array}{l}\text { Gorgulho- } \\
\text { do-milho }\end{array}$ & $\begin{array}{l}\text { Pulgão- } \\
\text { lanígero }\end{array}$ & $\begin{array}{l}\text { Piolho-de- } \\
\text { São-José }\end{array}$ & $\begin{array}{c}\text { Moscas-das- } \\
\text { frutas }\end{array}$ & $\begin{array}{l}\text { Grandes } \\
\text { lagartas }\end{array}$ & Sarna \\
\hline Testemunha & $4 a^{2}$ & $16 \mathrm{a}$ & $3^{\text {ns }}$ & $0^{\mathrm{ns}}$ & $7 \mathrm{a}$ & $60 \mathrm{a}$ & 0 & $24 \mathrm{~b}$ \\
\hline \multicolumn{9}{|c|}{ Sacos de papel-manteiga parafinado branco } \\
\hline $15 \mathrm{DAC}$ & $0 \mathrm{~b}$ & $7 \mathrm{ab}$ & 3 & 2 & $0 \mathrm{~b}$ & $26 \mathrm{~b}$ & 0 & $42 \mathrm{ab}$ \\
\hline $7 \mathrm{DAC}$ & $0 \mathrm{~b}$ & $4 \mathrm{~b}$ & 0 & 0 & $0 \mathrm{~b}$ & $6 \mathrm{c}$ & 0 & $38 \mathrm{ab}$ \\
\hline $\mathrm{DC}$ & $0 \mathrm{~b}$ & $7 \mathrm{ab}$ & 3 & 2 & $0 \mathrm{~b}$ & $4 \mathrm{c}$ & 0 & $36 \mathrm{ab}$ \\
\hline \multicolumn{9}{|c|}{ Sacos de polipropileno microperfurado transparente } \\
\hline $15 \mathrm{DAC}$ & $0 \mathrm{~b}$ & $5 \mathrm{~b}$ & 0 & 0 & $1 \mathrm{~b}$ & $18 \mathrm{bc}$ & 0 & $48 \mathrm{a}$ \\
\hline $7 \mathrm{DAC}$ & $0 \mathrm{~b}$ & $6 a b$ & 1 & 0 & $2 b$ & $12 \mathrm{bc}$ & 0 & $37 \mathrm{ab}$ \\
\hline DC & $0 \mathrm{~b}$ & $3 \mathrm{~b}$ & 1 & 0 & $0 \mathrm{~b}$ & $2 \mathrm{c}$ & 0 & $30 \mathrm{ab}$ \\
\hline
\end{tabular}

Testemunha sem proteção dos frutos; 15 DAC = 15 dias antes da colheita; 7 DAC = 7 dias antes da colheita; DC = dia da colheita.

${ }^{2}$ Médias seguidas de mesma letra na coluna não diferem estatisticamente entre si, pelo teste de Tukey, a $5 \%$ de probabilidade. ${ }^{\text {ns }}=$ não significativo pelo testeF, a $5 \%$ de probabilidade.

\section{MATERIAL E MÉTODOS}

O experimento foi realizado na safra 2004-2005, desenvolvidono pomar de produção orgânica da empresa Fischer Fraiburgo Agrícola Ltda. O pomar está localizado no município de , em Fraiburgo-SC (2701'43" S e 5055'17" W), região fisiográfica do Vale do Rio do Peixe, com altitude de $1.080 \mathrm{~m}$. na safra 2004/2005Descrever as características fisiográficas e climáticas da região. O clima da região é temperado constante úmido, com verão ameno, do tipo $\mathrm{Cfb}$, conforme a classificação de Köepen. O número médio de frio hibernal anual é de 600 horas $\leq 7,2^{\circ} \mathrm{C}$. A temperatura do ar e a precipitação total anual, média dos últimos anos, é de $16,1^{\circ} \mathrm{C}$ e $1.549 \mathrm{~mm}$, respectivamente (EPAGRI, 2002). As cultivares estudadas foram Royal Gala e Suprema, enxertadas sobre Marubakaido com filtro de M9. O pomar, com 4 anos de idade, apresenta 0,87 ha, com 2.360 plantas, no espaçamento de 4 $\mathrm{m}$ entre linhas e 1,25 m entre plantas. Cerca de $70 \%$ das plantas são de 'Royal Gala' e o restante de 'Suprema', distribuídas em linhas intercaladas. Os tratamentos foram compostos por três épocas de retirada dos sacos (15 e 7 dias antes da colheita e no dia da colheita); dois tipos de embalagens (saco de papel- manteiga parafinado branco, de $13,2 \mathrm{~cm} \times 22,2 \mathrm{~cm}$, e saco de polipropileno microperfurado transparente, de $17,0 \mathrm{~cm} \times 20,0 \mathrm{~cm}$ ); e a testemunha (frutos não ensacados). Ensacaram-se os frutos no dia 18-11-2004, quando estes apresentavam, em média, $2 \mathrm{~cm}$ de diâmetro. As avaliações da frutificação efetiva indicaram, em média, 12 frutos de 'Suprema' e 5 frutos de 'Royal Gala' por planta. Foram ensacados os frutos de 30 plantas de 'Royal Gala', totalizando 150 frutos por tratamento e 25 plantas de 'Suprema', totalizando 300 frutos por tratamento. Os frutos de 'Royal Gala' foram colhidos em 16-02-2005 e os de 'Suprema' em 18-03-2005. Em seguida, foram acondicionados em sacos plásticos e armazenados em câmara fria $\left(1^{\circ} \mathrm{C} \pm 1\right)$ até a data das avaliações. $\mathrm{Na}$ avaliação, foram utilizados 50 frutos de 'Royal Gala' e 100 frutos de 'Suprema' por tratamento, cada fruto constituindo uma repetição. Foram avaliados os danos causados pelos fatores bióticos: mariposa oriental (Grapholita molesta); lagarta-enroladeira (Bonagota cranaodes); gorgulho-do-milho (Sitophylus zeamais); pulgão-lanígero (Eriosoma lanigerum); cochonilha-piolho-de-São-José (Quadraspidiotus perniciosus); moscas-das-frutas (Anastrepha spp.); grandes lagartas e sarna (Venturia inaequalis), e os danos causados pelos fatores abióticos: "russeting" e queimadura pelo sol. Para as variáveis insetos, sarna e queimadura, avaliou-se o número de frutos com a presença ou a ausência destes danos na epiderme. Um caso especial foram os danos de pulgão-lanígero, que tem a característica de atacar raízes ou ramos da macieira. Neste caso, avaliou-se somente a presença do inseto no pedúnculo do fruto, ou seja, no ponto de inserção de ensacamento. Já para a avaliação do "russeting", adotouse uma escala, segundo as normas e padrões para classificação e comercialização de maçã (Brasil, 1980), sendo atribuídas notas quanto à percentagem deste distúrbio na epiderme dos frutos: $1=$ até $10 \%$ de "russeting" (tipo extra); $2=>10 \%$ e $<30 \%$ (tipo especial); $3=>30 \%$ e $<50 \%$ (tipo comercial), e $4=>50 \%$ (tipo indústria). Os resultados obtidos foram submetidos à análise de variância, determinando-se a significância, pelo teste F. Quando houve significância estatística, os tratamentos foram comparados entre si, pelo teste de Tukey, a 5\% de probabilidade.

\section{RESULTADOS E DISCUSSÃO}

\section{Danos de insetos-praga}

Os valores de danos causados pelos por insetos-praga encontram-se nas Tabelas 1 e 2. Na 'Royal Gala', não se observaram frutos danificados pela mariposa oriental e pela cochonilha-piolhode- São-José. Provavelmente, devido às épocas de ocorrência destes insetos dos, cujos picos populacionais destes insetos ocorreram após a colheita desta cultivar, que foi 30 dias antes da 'Suprema', resultando maior ocorrência de danos nesta última. Observou-se incidência esporádica de pulgão-lanígero na 'Suprema' e nula na 'Royal Gala'. A ausência de danos deste inseto-praga, certamente, está relacionada à resistência genética no porta-enxerto Marubakaido (Denardi, 2002). $\mathrm{O}$ ensacamento mostrou-se eficiente no controle da mariposa oriental na cv. Suprema, pois, observando-se danos somente nos frutos não ensacados tratamento testemunha. Assim como recomendado por Faoro (2003) para pereira japonesa, o uso do ensacamento de frutos é interessante para o controle deste inseto em maçã. Embora tenham sido observados danos de lagarta-enroladeira nas duas cultivares, o ensacamento mostrou-se efetivo somente na cv. Suprema em todas as épocas de retirada dos sacos. A presença de alguns frutos desensacados, no dia da colheita com danos de lagarta-enroladeira, pode ser devido ao rompimento de algumas embalagens, ocasionado pela chuva ou pelo granizo ocorridos no período. Os danos foram mais expressivos em frutos ensacados com sacos de papel-manteiga, devido esta embalagem ser menos resistente que a de polipropileno. Não houve diferença significativa entre o ensacamento ou não de frutos para os danos do gorgulho-do-milho em ambas as cultivares. Provavelmente, este inseto atacou frutos ensacados por apresentar tamanho bastante reduzido (aproximadamente $3 \mathrm{~mm}$ de comprimento), o que facilitou a sua entrada em sacos que não foram bem fechados. $\mathrm{Na}$ cv. Suprema, observou-se que houve diferença significativa entre o ensacamento ou não de frutos, em relação aos danos da cochonilhapiolho-de-São-José, onde os frutos não ensacados apresentaram mais danos deste inseto que os frutos ensacados. Devido à ocorrência esporádica de grandes lagartas, não foi possível avaliar o efeito do ensacamento sobre os danos causados por este grupo de insetospraga, não ocorrendo significância entre os tratamentos, nas duas cultivares. Nas duas cultivares, observou-se que houve diferença significativa de danos causados por moscas-das-frutas entre frutos 
TABELA 2- Percentagem de frutos danificados por fatores bióticos, em função do tipo de embalagem utilizada no ensacamento e da época de desensacamento - cv. Royal Gala. Fraiburgo-SC, safra 2004-2005.

\begin{tabular}{|c|c|c|c|c|c|c|c|c|}
\hline \multirow[b]{2}{*}{ Tratamento ${ }^{1}$} & \multicolumn{8}{|c|}{ Danos avaliados (\%) } \\
\hline & $\begin{array}{c}\text { Mariposa } \\
\text { oriental }\end{array}$ & $\begin{array}{c}\text { Lagarta- } \\
\text { Enroladeira }\end{array}$ & $\begin{array}{l}\text { Gorgulho- } \\
\text { do-milho }\end{array}$ & $\begin{array}{l}\text { Pulgão- } \\
\text { lanígero }\end{array}$ & $\begin{array}{l}\text { Piolho-de- } \\
\text { São-José }\end{array}$ & $\begin{array}{l}\text { Moscas-das- } \\
\text { frutas }\end{array}$ & $\begin{array}{l}\text { Grandes } \\
\text { lagartas }\end{array}$ & Sarna \\
\hline Testemunha & 0 & $6^{\mathrm{ns}}$ & $4^{\mathrm{ns}}$ & 0 & 0 & $20 a^{2}$ & $0^{\mathrm{ns}}$ & $40 \mathrm{ab}$ \\
\hline \multicolumn{9}{|c|}{ Sacos de papel-manteiga parafinado branco } \\
\hline $15 \mathrm{DAC}$ & 0 & 0 & 8 & 0 & 0 & $4 \mathrm{c}$ & 0 & $26 \mathrm{~b}$ \\
\hline 7 DAC & 0 & 4 & 2 & 0 & 0 & $4 \mathrm{c}$ & 0 & $56 \mathrm{a}$ \\
\hline DC & 0 & 2 & 2 & 0 & 0 & $2 \mathrm{c}$ & 0 & $50 \mathrm{ab}$ \\
\hline \multicolumn{9}{|c|}{ Sacos de polipropileno microperfurado transparente } \\
\hline $15 \mathrm{DAC}$ & 0 & 2 & 2 & 0 & 0 & $6 \mathrm{bc}$ & 0 & $36 \mathrm{ab}$ \\
\hline $7 \mathrm{DAC}$ & 0 & 0 & 2 & 0 & 0 & $8 \mathrm{bc}$ & 2 & $50 \mathrm{ab}$ \\
\hline DC & 0 & 6 & 4 & 0 & 0 & $4 \mathrm{c}$ & 0 & $50 \mathrm{ab}$ \\
\hline
\end{tabular}

${ }^{1}$ Testemunha sem proteção dos frutos; 15 DAC $=15$ dias antes da colheita; 7 DAC = 7 dias antes da colheita; DC = dia da colheita.

${ }^{2}$ Médias seguidas de mesma letra na coluna não diferem estatisticamente entre si, pelo teste de Tukey, a $5 \%$ de probabilidade. ${ }^{n s}=$ não significativo pelo teste-F, a $5 \%$ de probabilidade.

ensacados e não ensacados, sendo que os não-ensacados apresentaram mais danos deste inseto. Também houve o efeito da época de retirada dos sacos, onde a retirada próxima ao dia da colheita promoveu menor percentagem de frutos danificados por moscas-dasfrutas. Isto mostra a importância de se manterem os frutos ensacados por um maior período de tempo, principalmente pelo fato de as moscasdas-frutas serem a principal praga da cultura, promovendo maior percentagem de frutos danificados que os demais insetos.

\section{Dano de sarna}

A sarna desenvolveu-se em frutos ensacados e não ensacados (Tabela 1 e 2), provavelmente, em virtude de o patógeno já ter infectado o fruto antes do ensacamento. $\mathrm{O}$ ano foi bastante favorável para o desenvolvimento do patógeno, pois a primavera e o verão apresentaram temperaturas amenas e alta umidade, que, de acordo com Boneti et al. (2002), são fatores ideais para a doença. O ensacamento favoreceu o desenvolvimento do patógeno, principalmente na cv. Suprema quando os frutos permaneceram por um período maior no campo. Os sacos podem ter criado um microclima favorável para o desenvolvimento do patógeno. Já em 'Royal Gala', estes danos não foram tão expressivos entre os frutos não ensacados e ensacados.

\section{Dano de "russeting"}

Em média, os frutos das duas cultivares se enquadraram na classe "extra", que tolera para comercialização frutos com até $10 \%$ de "russeting". Houve diferenças significativas entre tratamentos para as duas cultivares (Tabela 3). De forma geral, houve mais frutos com "russeting", antecipando a retirada dos sacos. De acordo com Basso
(2002), a suscetibilidade ao "russeting" depende da cultivar, porém, do período da floração até um mês após, este distúrbio pode ser induzido por condições climáticas favoráveis, como temperaturas baixas e umidade no fruto.

\section{Dano de queimadura pelo sol}

Houve diferença significativa entre os tratamentos, somente para a cv. Suprema (Tabelas 3), visto que o ciclo desta cultivar é mais longo, coincidindo com período de maior incidência de radiação solar (meses de fevereiro e março). Além disso, a embalagem de polipropileno favoreceu este tipo de dano, principalmente quando se encontrava muito aderida à epiderme dos frutos (Tabelas 1).

\section{Praticidade das embalagens}

Verificou-se que o saco de polipropileno é mais fácil de ser manuseado, resultando em ensacamento mais rápido. Por ser transparente, esta embalagem facilita a entrada de raios solares, favorecendo o desenvolvimento de cor nos frutos. Além disto, caem menos, resistem mais à chuva e ao granizo, e o seu custo é menor em relação ao saco de papel-manteiga.

\section{CONCLUSÕES}

1) $\mathrm{O}$ ensacamento diminuiu o ataque de pragas em relação aos frutos não ensacados. Porém, a sarna se desenvolveu tanto em frutos ensacados quanto em não ensacados.

2) De forma geral, houve mais frutos com "russeting", antecipando a retirada dos sacos.

3) O saco de polipropileno é mais prático e mais resistente a

TABELA 3- "Russeting" em frutos (de acordo com escala) e percentagem de frutos com queimadura pelo sol, em função do tipo de embalagem utilizada no ensacamento e da época de desensacamento - cv. Suprema e Royal Gala. Fraiburgo-SC, safra 2004-2005.

\begin{tabular}{|c|c|c|c|c|}
\hline \multirow{3}{*}{ Tratamento $^{1}$} & \multicolumn{4}{|c|}{ Cultivar } \\
\hline & \multicolumn{2}{|c|}{ Suprema } & \multicolumn{2}{|c|}{ Royal Gala } \\
\hline & "Russeting"3 & $\begin{array}{c}\text { Queimadura pelo sol } \\
(\%)\end{array}$ & "Russeting" & $\begin{array}{c}\text { Queimadura pelo sol } \\
(\%)\end{array}$ \\
\hline Testemunha & $1,15 \mathrm{~b}^{2}$ & $1 \mathrm{~b}$ & $1,38 \mathrm{ab}$ & $0^{\mathrm{ns}}$ \\
\hline \multicolumn{5}{|c|}{ Sacos de papel-manteiga parafinado branco } \\
\hline 15 DAC & $1,35 \mathrm{a}$ & $0 \mathrm{~b}$ & $1,58 \mathrm{a}$ & 0 \\
\hline 7 DAC & $1,23 \mathrm{ab}$ & $0 \mathrm{~b}$ & $1,24 \mathrm{~b}$ & 0 \\
\hline DC & $1,08 \mathrm{~b}$ & $0 \mathrm{~b}$ & $1,38 \mathrm{ab}$ & 0 \\
\hline \multicolumn{5}{|c|}{ Sacos de polipropileno microperfurado transparente } \\
\hline $15 \mathrm{DAC}$ & $1,20 \mathrm{ab}$ & $11 \mathrm{a}$ & $1,30 \mathrm{ab}$ & 6 \\
\hline $7 \mathrm{DAC}$ & $1,17 \mathrm{~b}$ & $7 \mathrm{ab}$ & $1,18 \mathrm{~b}$ & 4 \\
\hline DC & $1,07 \mathrm{~b}$ & $10 \mathrm{a}$ & $1,22 \mathrm{~b}$ & 2 \\
\hline
\end{tabular}

Testemunha sem proteção dos frutos; 15 DAC $=15$ dias antes da colheita; 7 DAC $=7$ dias antes da colheita; DC $=$ dia da colheita.

${ }^{2}$ Médias seguidas de mesma letra na coluna não diferem estatisticamente entre si, pelo teste de Tukey, a 5\% de probabilidade. ${ }^{\text {ns }}=$ não significativo pelo teste

$\mathrm{F}$, a $5 \%$ de probabilidade.

${ }^{3}$ Notas quanto à percentagem de "Russeting" na epiderme dos frutos: $1=$ até $10 \%$ de "russeting" (tipo extra); $2=>10 \%$ e $<30 \%$ (tipo especial); $3=>30 \%$ e $<50 \%$ (tipo comercial) e $4=>50 \%$ (tipo indústria). 
intempéries que o saco de papel- manteiga. Porém, há maior insolação nos frutos, resultando em mais queimaduras, especialmente quando o saco se encontra muito aderido à epiderme do fruto.

\section{AGRADECIMENTOS}

Os autores agradecem aos funcionários da empresa Fischer Fraiburgo Agrícola Ltda., pelo auxílio no ensacamento dos frutos e aos pesquisadores da Estação Experimental da Epagri de Caçador M.Sc. Frederico Denardi e Ph.D. Clori Basso, pelo auxílio na revisão deste trabalho.

\section{REFERÊNCIAS}

ABPM. Informações Estatísticas. Disponível em: <http:// www.abpm.org.br/informações.htm> . Acesso em: 20 dez.2003.

ABPM. Informações estatísticas. Disponível em: <http:// www.abpm.org.br/informações.htm>. Acesso em: 10 dez.2004.

BASSO, C. Distúrbios fisiológicos. In: EPAGRI. A cultura da macieira. Florianópolis: Epagri, 2002. p.609-636.

BRASIL. Ministério da Agricultura. Secretaria Nacional do Abastecimento. Normas e padrões de identificação, qualidade, embalagem, classificação e comercialização de maçã. Brasília, 1980.20p.

BONETI, J.I.S; KATSURAYAMA, Y.; BLEICHER, J. Doenças da macieira. In: EPAGRI. A cultura da macieira. Florianópolis: Epagri, 2002.p.527-608
CAMILO, A.P.; DENARDI, F. Cultivares: Descrição e comportamento no sul do Brasil. In: EPAGRI. A cultura da macieira. Florianópolis: Epagri, 2002. p.113-168.

DENARDI, F. Porta-enxertos. In: EPAGRI. A cultura da macieira. Florianópolis: Epagri, 2002. p.169-227.

FAORO, I.D. Raleio e ensacamento. In: CURSO SOBRE A CULTURA DA PEREIRA, 2., 2000, Caçador. Trabalhos apresentados...Caçador: Epagri/Jica, 2000. p. 47-52.

FAORO, I.D.; YASUNOBU, Y. Investigação dos fatores de necrose de gemas e seu controle. In: PROJETO PESQUISA EM HORTICULTURAPARAPEQUENOS PRODUTORES NOSULDO BRASIL. São Joaquim-SC: Epagri/Jica/Embrapa, 2001 (Relatório Anual 2000-2001).

FAORO, I.D. Técnica e custo para o ensacamento de frutos de pêra japonesa. Revista Brasileira de Fruticultura, Jaboticabal, v.25, n.2, p.339-340, 2003.

IBGE. Disponível em: <http://www.abpm.org.br/informações.htm>. acesso em: 20 dez.2004.

PANDOLFO, C. (Coord.). BRAGA, H.J.; SILVA JÚNIOR, V.P.; MASSIGNAM, A.M.; PEREIRA, E.S.; THOMÉ, U.M.R.. Atlas climatológico do estado de Santa Catarina. Florianópolis: Epagri, 2002. CD-ROM.

TELLES, C.A.; BIASI, L.A.; RIBEIRO, A.N.; MASCHIO, P.A. Produção e qualidade de pêssegos ensacados da cultivar Coral. Revista Agropecuária Catarinense, Florianópolis, v.17, n.1, p.83-86, 2004. 